\title{
Influence of Site Parameters on Fourier Amplification Application for 1D Linear Viscoelastic Method
}

\author{
Ahmed Boudghene Stambouli ${ }^{1 *}$, Djawad Zendagui ${ }^{1}$, Pierre-Yves Bard ${ }^{2}$, Hamza Dif ${ }^{1,3}$ \\ ${ }^{1}$ Risk Assessment and Management Laboratory (RISAM), Faculty of Technology, University of Tlemcen, BP 230-13048, Tlemcen, Algeria \\ ${ }^{2}$ Institut de Sciences de la Terre (ISTerre), Université Grenoble-Alpes, IFSTTAR, Maison des Géosciences, Bâtiment OSUG C, CS 40700 , \\ 38058 GRENOBLE Cedex 9, France \\ ${ }^{3}$ Département de Génie Civil, Université de Djelfa, Faculté de Technologie, BP 3117, Djelfa, Algeria \\ * Corresponding author, e-mail: ahmed-boudghene-stambouli@univ-tlemcen.dz
}

Received: 29 May 2018, Accepted: 28 September 2020, Published online: 04 November 2020

\begin{abstract}
We focus on the effect of site parameters, also called site proxies, on the variation of the amplification factor. This latter, named Fourier Amplification Factor (FAF) is defined as the ratio of the Fourier transform of the seismic motion at surface and at bedrock. For this study, the wave propagation theory is used limited to 1D linear viscoelastic domain. At this effect, a set of FAF, is established for a set of 858 real profiles. From there, the site parameters are also derived, it is necessary to mention that the FAF can be computed in independent manner of seismic signals which it is applicable only on linear domain.

In Nuclear Power Industry application, the FAF is mostly used and can be approximated by limited number of site proxies. As the usual code practice implies a lower number of site proxies (generally 1, sometimes 2) as UBC97 or EC8, a sensitivity analysis is conducted to identify the "best performing" site parameters. The results show that by far, using the six site proxies lead to a better prediction of FAF. However if we have to use one single site parameter, results show that the best one is the overall resonance frequency $\left(f_{0}\right)$. In the case when we intend to use two site parameters built from the average shear wave velocity over the upper $30 \mathrm{~m}\left(V_{s 30}\right)$ and the resonance frequency $\left(f_{0}\right)$ which are preferred and give an important variance reduction superior than $61 \%$. In the result, a new formula has been established.
\end{abstract}

Keywords

1D linear site response, Fourier amplification factors, site proxies, neural network

\section{Introduction}

Seismic site effect is perhaps one of the most studied phenomena during the last decades. Trifunac [1] states that "Investigations of the effects of local site conditions on the characteristics of strong earthquake ground motion are probably as old or even older than the discipline of Earthquake Engineering itself". Since the earlier works such as: the pointing work of Thomson [2] and Haskell [3], many works and papers have been published to describe the site effect. The most common approach used to assess site effect is to compare a seismic parameter (acceleration, velocity, etc.) obtained at free surface and at bedrock. For instance, pioneer work used the peak ground acceleration (PGA) as seismic parameter. The amplification factor is hence defined as the ratio of PGA at free surface and at bedrock.

However, it is admitted that the seismic action could not be captured by one scalar value, in addition to the peak value of the seismic motion, its frequency content could have great influence on site effect $[4,5]$. In this study, the site effect is assessed through the Fourier transform of the seismic motion which is mostly used in Nuclear Power Industry (see for instance: EPRI [6]; The US Nuclear Regulatory Commission (USNRC) [7]; Ktenidou and Abrahamson [8]; Bard et al. [9]), in contrary to civil engineering application which is based on the response spectra (e. g: UBC97; EC8;...) $[10,11]$. In a separate work of Boudghene Stambouli et al. [12], the response spectra has been used to assess the site effect.

Many authors were interested in the evaluation of Fourier amplification ratio, for example the work of Andrews [13] in reference site approach which developed generalized inversion using Fourier spectra of observed motions where they use geometric spreading to evaluate source and site 
terms simultaneously. The goal of this latter is to allow having an arbitrary frequency dependence and the site response terms are taken as relative to the network average [13] or relative to a single pre-determined "reference" site, which the site term is unity (e.g., Boatwright et al. [14]; Hartzell [15]). Another authors, like Sokolov [16] and Sokolov et al. [17] derive locally the attenuation functions Fourier amplitude spectra to specific sedimentary basin. And for almost all recent ground-motion prediction equations (GMPEs) developing empirical prediction equations for Fourier spectra on a particular duration estimated of ground motion using random vibration theory application for seismic ground motion recorded in Europe (RESORCE-2012) (see for instance: Bora et al. [18-20]).

In this paper, the amplification factor is defined as the difference or the ratio of the Fourier transform of the seismic motion between free surface and bedrock using wave propagation theory limited to linear domain which is independent to the seismic loading. For this context, it is necessary to mention that, the application of 1D linear viscoelastic are limited to low seismicity areas which, the peak ground acceleration (PGA) in the bedrock do not exceed $0.2 \mathrm{~g}$ (see for instance: O'Connell [21]). And more other authors are demonstrating this precise point, like Trifunac and Todorovska [22] which deduced that the 1D linear site response is satisfactory for the peak ground velocity (PGV) less than 30 gal (corresponding to PGA around to $0.2 \mathrm{~g}$ ) and also for more recent study is approved in a real case application for 1980 El-Asnam earthquake (see for instance: Cherid et al. [23]).

This amplification is named hereafter Fourier Amplification Factor (FAF).

Nowadays, many codes like SHAKE (Schnabel [24]), EERA(Bardet et al. [25]) or DEEPSOIL (Hashash et al. [26]) provide the FAF in term of a transfer function in the linear and non-linear domain. The user of this software introduces the mechanical and geometrical properties of the soil layer together with the seismic excitation at the basement. Although these codes allow the assessment of FAF, it is desirable to have a practical tool that gives an idea on how the soil properties shape the form of the FAF, which is mostly used in Nuclear power industry application. The main idea is to derive FAF from simple to use site parameters. From where do we derive these site parameters? This paper uses site parameters (called also site proxies) from the soil properties (both mechanical and geometrical).

Thus, this paper addresses the influence of these site proxies on the variation of FAF.
For this purpose a total of 858 real soil profiles are considered(as using by Boudghene Stambouli et al. [12]), and their linear visco-elastic response to vertically incident $\mathrm{S}$ waves is computed for any real input waveforms spanning a wide range of frequency contents. The soil profile that belongs to this serie is named Real Profile (REP). For each site, FAF is derived from 200 values of frequency equally spaced between $0.01 \mathrm{~Hz}$ and $50 \mathrm{~Hz}$ on a logarithmic axis and at the resonant frequency. It is important to note that the bedrock velocity for the 858 soil profiles is not the same and sometimes reaches the value of $2 \mathrm{~km} / \mathrm{s}$. In order to have a uniform bedrock velocity we have established a second series of soil profile. Soil profile that belongs to this serie is named Truncated Profile (TRP). In order to measure the effect of site proxies on FAF, a neural network called Generalized regression neural network (GRNN) has been used. The huge quantity of information has been used to feed the neural network. Through this strategy we will measure the influence of each site parameters on the variation of FAF. Finally, the Fourier amplification factor corresponding to resonance frequency for any sites has been established using simple site proxies describing by $V_{s 30}$ and $f_{0}$ in input.

\section{Derivation of amplification factors FAF 2.1 Overall procedure}

The goal of this section is to describe the procedure to obtain a set of FAF for all soil profiles. Basically, for a particular soil profile, the FAF is computed as the ratio between acceleration on soil surface and outcropping reference rock in Fourier domain. However in 1D linear visco-elastic analysis, the FAF can be computed as the module of the transfer function without prior information on time history acceleration (see for instance: Kramer [27]). Thus:

$$
F A F(f)=a b s(T(f)),
$$

where $T(f)$ is the corresponding transfer function for a soil profile using $1 \mathrm{D}$ linear visco-elastic analysis and $a b s\left({ }^{*}\right)$ is the absolute value operator.

In this study, we consider that the soil is composed of (n) horizontally layered soils deposit resting on substratum and subjected to vertically shear waves (Fig. 1).

Each layer $(m)$ is fully known by its shear modulus $\left(G_{m}\right)$, thickness $\left(h_{m}\right)$, mass density $\left(\rho_{m}\right)$ and damping ratio $\left(\xi_{m}\right)$. The soil profile is subjected to a vertically shear wave which causes horizontal deformation governed by the following equation $[2,7]$ : 


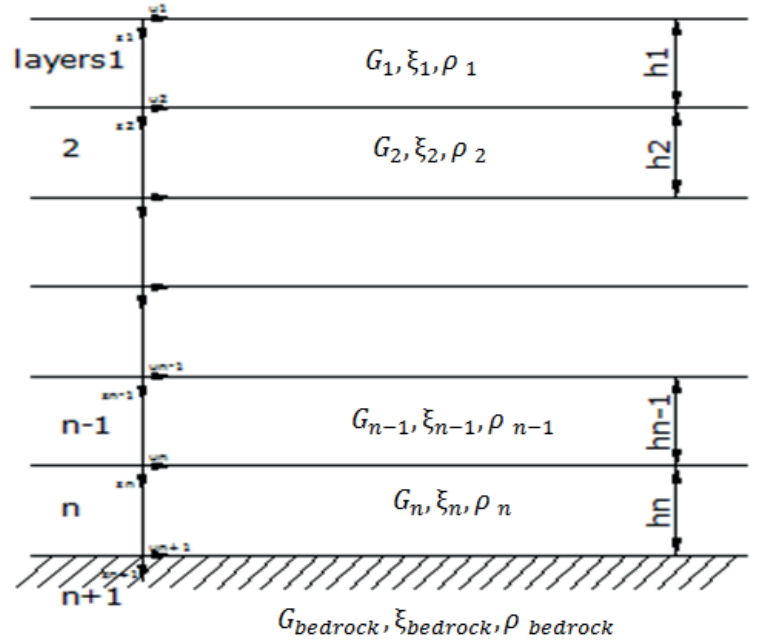

Fig. 1 General description of a horizontally layered soil deposit

$G_{m} \frac{\partial^{2} u_{m}}{\partial z_{m}^{2}}+_{m} \frac{\partial^{3} u_{m}}{\partial z_{m} \partial t}=\rho_{m} \frac{\partial^{2} u_{m}}{\partial t^{2}}$,

where: $\eta_{m}=2 G_{m} \xi_{m} / \omega$

The damping ratio is defined as follow:

$\zeta_{m}=\frac{1}{2 Q_{m}}$,

which the quality factor $Q_{m}$ is described by (Aki and Richards [28], Fukushima et al. [29], Boudghene Stambouli et al. [12]):

$Q_{m}=V_{m} / 10$,

hence

$\zeta_{m}=\frac{5}{V_{m}}$.

For a particular point in layer $(m)$, the solution of the wave equation could be obtained [27]:

$u_{m}\left(z_{m}, f\right)=A_{m} e^{(i 2 \pi f t)} e^{\left(i k_{m}^{*} z_{m}\right)}+B_{m} e^{(i 2 \pi f t)} e^{\left(-i k_{m}^{*} z_{m}\right)}$.

The transfer function could be obtained as follow:

$T(f)=u_{1}\left(z_{1}=0, f\right) / u_{n+1}\left(z_{n+1}=0, f\right)$.

The FAF could be determined by using Eqs. (1)-(7). Although popular codes like DEEPSOIL [26] and EERA [25] provide transfer function, we developed our own code since the FAF will be calculated for hundred soil profiles. This code has been successfully checked against DEEPSOIL and EERA. For instance considering a single layer with a shear wave velocity of $200 \mathrm{~m} / \mathrm{s}$ and thickness of $30 \mathrm{~m}$ resting on bedrock with shear velocity of $800 \mathrm{~m} / \mathrm{s}$.

\subsection{Soil profiles and determination of site parameters}

\subsubsection{Description of used soil profile}

In this study we will consider a database composed of $n_{P}$ soil profiles. Each soil profile is fully described by it mechanical and geometrical properties (Fig. 1). A total of 858 real soil columns are considered about (600 Japanese KiK-net sites, more than 200 sites from the USA [12], and 22 European sites measured during the NERIES project (Di Giulio et al. [30]).

Obviously the shear wave velocity at the substratum will not be the same for all soil profiles. This velocity could reach high values and will be somehow in contradiction with regulation code which consider that the highest value of this velocity could not exceed a common value of $800 \mathrm{~m} / \mathrm{s}$. For this purpose we will have two databases (a) the first one is composed of the initial soil profile and will be labeled Real profile (REP) (b) the second one is composed of Truncated profile (TRP) which is characterized by a common shear wave velocity at the substratum. How do we establish the TRP database from the REP database? Let consider a soil profile that belongs to the REP database. Consider for instance that this soil profile consists of three layers resting on a bedrock: Layer $1\left(V_{1}=200 \mathrm{~m} / \mathrm{s}\right.$, $\left.h_{1}=5 \mathrm{~m}\right)$, Layer $2\left(V_{2}=600 \mathrm{~m} / \mathrm{s}, h_{2}=10 \mathrm{~m} / \mathrm{s}\right)$, Layer 3 $\left(V_{3}=800 \mathrm{~m} / \mathrm{s}, h_{3}=15 \mathrm{~m}\right)$ and $V_{\text {bedrock }}=1200 \mathrm{~m} / \mathrm{s}$, so the truncated soil profile will be composed of only two layers resting on a bedrock: Layer $1,\left(V_{1}=200 \mathrm{~m} / \mathrm{s}, h_{1}=5 \mathrm{~m}\right)$, Layer $2\left(V_{2}=600 \mathrm{~m} / \mathrm{s}, h_{2}=10 \mathrm{~m}\right)$ and $V_{\text {bedrock }}=800 \mathrm{~m} / \mathrm{s}$.

\subsubsection{Determination of site parameters}

Each soil profile (such as: REP or TRP) could be described by a set of simple parameters called hereafter "site parameters". This set of parameters has been fully described by Boudghene Stambouli et al. [12]. They are a total of 6 : a total depth until bedrock (Depth), the average shear velocity $\left(V_{s m}\right)$ over that Depth (subscript $s m$ stands for shear wave velocity mean value), average shear wave velocity of the upper $30 \mathrm{~m}\left(V_{s 30}\right)$, the shear wave velocity of the bedrock $\left(V_{\text {bedrock }}\right)$, the ratio between shear wave velocity of the bedrock and shear wave velocity at the surface $\left(C_{v}\right)$ and the fundamental frequency of soil profile $\left(f_{0}\right)$. It is important to note that a TRP is characterized by only five parameters since the shear wave velocity of the bedrock is constant and equal to $800 \mathrm{~m} / \mathrm{s}$. The number of site parameters for TRP is $R=5$ and for REP $R=6$.

A set of $n_{P}=858$ soil profiles corresponding to real sites was considered in this work. The distribution of site parameters could be found in Boudghene Stambouli et al. [12]. 


\section{Resulting set of FAF: main statistical characteristics}

\subsection{General background}

The FAF (Eq. (1)) has been calculated for soil profile that belongs to either REP or TRP database. They may be written as $\operatorname{FAF}\left(P_{k}, \theta, f_{i}\right)$, where :

$P_{k}\left(k=1, \ldots, n_{P}\right)$ is introduced to identify the soil profile. Note that $n_{P}=858$.

$\theta=1$ for soil that are labeled 'REP' and $\theta=2$ for soil that are labeled 'TRP'.

$f_{i}$ is the $i$ th frequency $(i=1, \ldots, 200)$. Actually FAF is systematically computed for 200 values, equally spaced between $0.01 \mathrm{~Hz}$ and $50 \mathrm{~Hz}$ on a logarithmic frequency axis. For instance $\operatorname{FAF}\left(P_{20}, 2, f_{20}\right)$ stands for the FAF obtained at $f_{20}$ for the truncated soil profile $P_{20}$. As we have $n_{P}$ FAF we will derive a mean value and the variability of the $n_{P}$ FAF. Hence we have the:

- Average of FAF over all profiles, noted $\operatorname{FAF}\left(\theta, f_{i}\right)$ which it is computed from Fourier amplification $\operatorname{FAF}\left(P_{k}, \theta, f_{i}\right)$ for all profiles:

$$
\log \left(F A F\left(\theta, f_{i}\right)\right)=\frac{1}{n_{p}} \sum_{k=1}^{n_{p}}\left[\log \left(F A F\left(P_{k}, \theta, f_{i}\right)\right)\right] .
$$

- Initial variability is the standard deviation of the site Fourier amplification factor over all profiles:

$$
\sigma\left(\theta, f_{i}\right)=\sqrt{\frac{1}{n_{p}} \sum_{k=1}^{n_{p}}\left[\log \left(F A F\left(P_{k}, \theta, f_{i}\right)\right)-\log \left(F A F\left(\theta, f_{i}\right)\right)\right]^{2}} .
$$

- Maximum initial variability, defined as the maximum over frequencies of initial variability

$$
\sigma_{\max }(\theta)=\max \left[\sigma\left(\theta, f_{i}\right)\right] .
$$

- Overall initial variability, defined as the average over all frequencies of the initial variability $\sigma\left(\theta, f_{i}\right)$ :

$$
\sigma_{m}(\theta)=\frac{1}{n_{f}} \sum_{i=1}^{n_{f}} \sigma\left(\theta, f_{i}\right) \text {. }
$$

Where is the number of frequencies used, i.e., 200.

\subsection{Variability of FAF}

We have computed $n_{P}$ FAF for the REP and TRP, $\operatorname{FAF}\left(P_{k}, \theta, f_{i}\right), \operatorname{FAF}\left(\theta, f_{i}\right)$ and $\sigma\left(\theta, f_{i}\right)$. Fig. 2(a) exhibits the variation of the $n_{P} \operatorname{FAF}\left(P_{k}, 1, f_{i}\right)$ (continuous line) and $\operatorname{FAF}\left(1, f_{i}\right)$ (dot line). Fig. 2(b) exhibits the variation of the $\operatorname{FAF}\left(P_{k}, 2, f_{i}\right)$ (continuous line) and $\operatorname{FAF}\left(2, f_{i}\right)$ (dot line). Careful examination of Fig. 2 shows that:

The peak frequency (i.e., the frequency with maximum amplification) covers a broad range, from $7 \mathrm{~Hz}$ to about $10-12 \mathrm{~Hz}$
The corresponding peak value of FAF ranges from less than 1.5 up to 15.

On the other hand we have measured the corresponding "initial variability" (Table 1). We found that it reaches its maximum at high frequency.
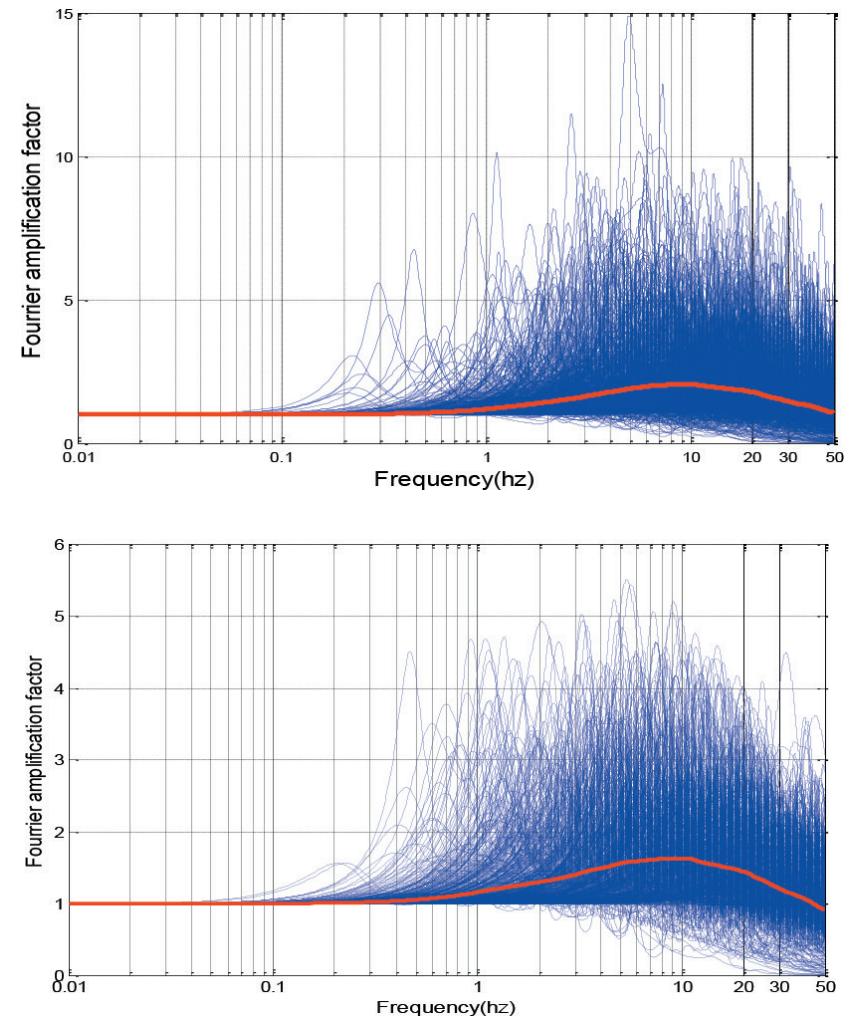

Fig. 2 Average $\operatorname{FAF}\left(P_{k}, \theta, f_{i}\right)$ for all soil profiles, together with their average $\operatorname{FAF}\left(\theta, f_{i}\right)$ (red line) for REP (top), and TRP (bottom)

Table 1 Initial variability values for the Fourier amplification factors for the REP and TRP profile sets

\begin{tabular}{lcc}
\hline Initial variability & $\theta=1$ & $\theta=2$ \\
\hline$\sigma(\theta, f=0.015 \mathrm{~Hz})$ & 0.0002 & 0.0001 \\
$\sigma(\theta, f=0.04 \mathrm{~Hz})$ & 0.0012 & 0.0007 \\
$\sigma(\theta, f=0.01 \mathrm{~Hz})$ & 0.0073 & 0.0041 \\
$\sigma(\theta, f=0.15 \mathrm{~Hz})$ & 0.0167 & 0.0088 \\
$\sigma(\theta, f=0.2 \mathrm{~Hz})$ & 0.0284 & 0.0138 \\
$\sigma(\theta, f=0.35 \mathrm{~Hz})$ & 0.0454 & 0.0317 \\
$\sigma(\theta, f=0.5 \mathrm{~Hz})$ & 0.0597 & 0.0537 \\
$\sigma(\theta, f=1 \mathrm{~Hz})$ & 0.109 & 0.1039 \\
$\sigma(\theta, f=5 \mathrm{~Hz})$ & 0.2045 & 0.1614 \\
$\sigma(\theta, f=10 \mathrm{~Hz})$ & 0.2203 & 0.1628 \\
$\sigma(\theta, f=15 \mathrm{~Hz})$ & 0.2349 & 0.1685 \\
$\sigma(\theta, f=20 \mathrm{~Hz})$ & 0.2575 & 0.1886 \\
$\sigma(\theta, f=30 \mathrm{~Hz})$ & 0.2862 & 0.2205 \\
$\sigma(\theta, f=40 \mathrm{~Hz})$ & 0.3334 & 0.2697 \\
$\sigma(\theta, f=50 \mathrm{~Hz})$ & 0.3827 & 0.323 \\
\hline
\end{tabular}


The database in terms of FAF, ie $\operatorname{FAF}\left(P_{k}, \theta, f_{i}\right)$, has been constituted for REP and TRP soil profiles. Note that as $f_{i}$ is not generally equal to $f_{0}$ then we have constituted the database in terms of $\operatorname{FAF}\left(P_{k}, \theta, f_{0}\right)$.

The main issue now is to understand how the site parameters shape the value of FAF to different value of frequency and for the fundamental soil frequency. To reach this goal, we have used a newly neural proposed network approach called Generalized Regression Neural Network (GRNN). Next section is dedicated to a short description of this GRNN approach and how we will implement it.

\section{Short description of the neural network used 4.1 Description of Generalized Regression Neural Network (GRNN)}

In 1991, Specht proposed a new version of neural network named generalized regression neural network (GRNN) [31]. GRNN can construct a good approximation of any continuous function which is approximated by a linear combination of well-chosen Gaussian functions. The GRNN is a variation of radial basis neural networks (RBF) (see for instance: Kim et al. [32]), It is based on kernel regression networks where it has been derived from statistics independently in the first by Nadaraya [33] and Watson [34] using mathematical foundation given by Parzen on kernel density estimation (see for instance: Parzen [35]). One of the powerful of this method is in the training phase which we have only the width of the Gaussian to adjust in contrary of Multi-Layer Perceptron (MLP) which is used a procedure as back propagation networks (see for instance: Benahmed et al. [36]).

The topology or the architecture of GRNN consist on 4 layer (see for instance: Fig. 3), it is described as: input, pattern, summation and output layer.

- The input layer consists of the values of the site parameters taken $\left(x_{k}\right)$ (in this study are $6(k=6)$ for real profiles (REP) and are $5(k=5)$ for truncated profiles (TRP)).

- The pattern layer is the processing unit which is composed of neurons, each of these neurons possesses an activation function described by the Gaussian $\left(\exp \left(-b^{2}\left(D_{t}\right)^{2}\right)\right)$.In this layer the weight is computed by the Euclidian distance $\left(D_{t}\right)$ between the input (site parameters taking $\left(x_{k}\right)$ ) and the training set $\left(I W_{t, k}\right)$ generally adjusted by bias $(b)$ (see for instance: Wasserman [37]). The result of this layer is described by Eq. (12):

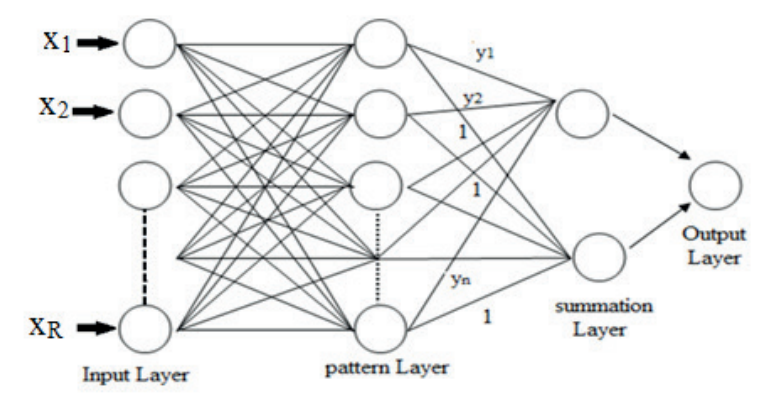

Fig. 3 General architecture of a neural network in the GRNN approach $w_{t}=e^{-\left(\text {Dist }_{t}^{*} b\right)^{2}}$.

With: Dist $_{t}=I W-x=\sqrt{\sum_{k=1}^{R}\left(I W_{t, k}-x_{k}\right)^{2}}$

The number of neurons in this layer is equal to numbers of profiles submitted to the learning phase.

- The third layer is the summation layer consisting of two neurons corresponding to two types of summation $S$ and $D$.

- $S$ summation neuron does the matrix multiplication between the matrix weight $y$ and the output of pattern layer $(w)$, the following equation can be expressed such as: $S=y^{\prime} * w$.

- $y$ the matrix weight between pattern and summation layer which is determined automatically in training phase,

- ' indicating the transpose of the matrix weight, $w$ is the vector output of pattern layer, the symbol * indicate the matrix multiplication.

- $D$ summation neurons perform the sum of the result come from pattern layer $D=\sum_{t=1}^{Q} w_{t}$.

- The output or the exit layer consists to perform the division of $S$ by $D$ (see for instance: Specht [32]; Jang et al. [41]), the equation can be described such as:

$$
\text { result }=y^{*} w / \sum_{t=1}^{n} w_{t} .
$$

- $n$ : is the number of neurones in pattern layers.

- $x_{k}$ : the $k$ th component of the input $\mathbf{x}$ vector of dimension $R$, for which the output is seeked. For our case this vector contains the 6 site parameters defined in the section above ( $R=6$ for REP, or $R=5$ for TRP). Thus for soil that belongs to REP.

$$
\begin{aligned}
& \{X\}^{T}=\left\{\begin{array}{llllll}
x_{1} & x_{2} & x_{3} & x_{4} & x_{5} & x_{6}
\end{array}\right\}^{T} \\
& =\left\{\begin{array}{lllllll}
\text { Depth } & V_{s m} & V_{s 30} & C_{v} & f_{0} & V_{\text {bedrock }}
\end{array}\right\}^{T}
\end{aligned}
$$


Note that for soil that belong to TRP, the last input $x_{6}=V_{\text {bedrock }}$ is not included

$y_{t}$ is the weight of the connection between the th neuron of the pattern layer ( $=t$ th learning site) and the S-summation neuron.

$x_{t k}$ the learning vector between the $t$ th neuron of the hidden layer and the entry vector of the $\mathrm{K}$ line, corresponding to the value of the $k$ th site parameter for the learning site $t$,

$b$ the bias between input and pattern layer which it is function on Gaussian width.

$n$ is the number of vectors submitted to the learning.

$R$ is the size of the input vector which is the number of site parameters to be used as an input.

\subsection{Use of the implementation}

As we have two set of soil profile namely REP and TRP, the implementation will be done separately. For each case, the initial set of data to feed the neural network is constituted by a subset of a combination of the site parameters ( 6 for the REP, and 5 for TRP) on one hand, and on the other hand the corresponding amplification factors $\operatorname{FAF}\left(P_{k}, \theta, f_{i}\right)$ or $\operatorname{FAF}\left(P_{k}, \theta, f_{0}\right)$. It is obvious that we can have many combinations in terms of the number and type of site parameters. For instance, if we consider only one parameter then for REP we will have six possibilities whereas for TRP we will have only five. Let consider the case of two parameters. For soil that belongs to REP, we thus have fifteen combinations (see for instance: Table 2). Again let consider a soil that belongs to REP, if we consider six parameters then we will have only one combination.

The output consists of the calculated FAF for a given frequency (200 values). There is one GRNN model for each frequency, i.e., 200 scalar models for $\operatorname{FAF}\left(P_{k}, \theta, f_{i}\right)$. This output FAF depends on the combination of site parameters and will be referred to $\operatorname{asAF}\left(P_{k}, \theta, f_{i}, x_{1}, \ldots, x_{R}\right)$, where

$$
\begin{aligned}
& \left\{\begin{array}{llllll}
x_{1} & x_{2} & x_{3} & x_{4} & x_{5} & x_{6}
\end{array}\right\}^{T} \\
& =\left\{\begin{array}{lllllll}
\text { Depth } & V_{s m} & V_{s 30} & C_{v} & f_{0} & V_{\text {bedrock }}
\end{array}\right\}^{T}
\end{aligned}
$$

For instance let consider the soil profile $P_{200}$ that belongs to the REP, then the computed FAF is $\operatorname{FAF}\left(P_{200}, 1, f_{i}\right)$. Consider that we will introduce two site parameters (let say $x_{4}=C_{v}$ and $\left.x_{6}=V_{\text {bedrock }}\right)$. Then the initial set of data to feed the neural network is constituted of $\operatorname{AF}\left(P_{200}, 1, f_{i}\right)$ and the pair $\left(C_{v}, V_{\text {bedrock }}\right)$. The output will be then $\operatorname{FAF}\left(P_{200}, 1, f_{i}, x_{4}, x_{6}\right)$ which is considered as the FAF deduced from the GRNN model.

\begin{tabular}{|c|c|c|c|c|}
\hline $\begin{array}{c}\text { Set of } \\
\text { Profiles }\end{array}$ & $\begin{array}{c}\text { Set of } \\
\text { explanatory } \\
\text { variables (Site } \\
\text { parameters) }\end{array}$ & $\begin{array}{c}\text { Type of } \\
\text { combination }\end{array}$ & $\begin{array}{l}\text { Number of } \\
\text { combination }\end{array}$ & $\begin{array}{c}\text { Total } \\
\text { number of } \\
\text { combination }\end{array}$ \\
\hline \multirow{6}{*}{ REP } & \multirow{6}{*}{$\begin{array}{c}6: \text { Depth }, f_{0} \\
C_{v}, V_{s m}, V_{s 30} \\
V_{\text {bedrock }}\end{array}$} & 1-parameter & 6 & \multirow{6}{*}{63} \\
\hline & & 2-parameter & 15 & \\
\hline & & 3-parameter & 20 & \\
\hline & & 4-parameter & 15 & \\
\hline & & 5-parameter & 6 & \\
\hline & & 6-parameter & 1 & \\
\hline \multirow{5}{*}{ TRP } & \multirow{5}{*}{$\begin{array}{c}5: \text { Depth, } f_{0} \\
C_{v}, V_{s m}, V_{s 30}\end{array}$} & 1-parameter & 5 & \multirow{5}{*}{31} \\
\hline & & 2-parameter & 10 & \\
\hline & & 3-parameter & 10 & \\
\hline & & 4-parameter & 5 & \\
\hline & & 5-parameter & 1 & \\
\hline
\end{tabular}

Table 2 List of all considered GRNN models

The neural networks are trained by dividing the dataset into two datasets, a training set $(50 \%)$ and a testing set $(50 \%)$, the elements of which are randomly swapped from one set to another until obtaining the width of the Gaussian.

The performance of the GRNN model through various non-independent indicators such as the coefficient of correlation, the standard deviation of residuals, the reduction of standard deviation and variance reduction with respect to the initial variability.

\section{Results and discussion}

\subsection{Comparisons between FAF deduced from GRNN and analytical model}

The goal is to test the ability of the GRNN models using the site parameters to predict the FAF. Globally we intend to compare $\operatorname{FAF}\left(P_{k}, \theta, f_{i}\right)$ and $\operatorname{FAF}\left(P_{k}, \theta, f_{i}, x_{1}, \ldots, x_{R}\right)$.

To achieve the above mentioned comparison, we derived a large models of GRNN using all possibilities of combinations as input parameters, and we analyzed the evolution of the standard deviation of residuals (predicted value-actual values), also comparing it with the initial and the overall variability defined above.

Considering the various sets of site parameters as listed in Table 2 a total of 188 GRNN models was thus obtained (For all frequencies, and FAF which correspond to resonance frequency) and their performance are analyzed in a systematic way through the values of residuals. Fig. 4 gives the FAF predicted by a few GRNN models to the actual FAF (computed from the full 1D soil column) for two soil profiles (see Table 3). The first one corresponds to 
one of the REP sites, the other corresponds to TRP but not one of the TRP used in the training phase. Site parameters of both soil profiles are depicted in Tables 3 and 4 .

We show results for only few GRNN models since we have an important number of combinations (for instance for REP we have 63 combinations) and our goal is to show that by increasing the number of site parameters the FAF predicted by GRNN model is close to the FAF deduced from analytical one. For the sake of explanation, we will hereafter define the corresponding function of curves in let say Fig. 4 (top): Target outputs $=\operatorname{FAF}\left(P_{100}, 1, f_{i}\right)$

GRNN outputs (Inputs all parameters) $=$

$\operatorname{FAF}\left(P_{100}, 1, f_{i}, x_{1}, x_{2}, x_{3}, x_{4}, x_{5}, x_{6}\right)$

GRNN outputs (Input $C_{v}, V_{s 30}, f_{0}$ ) $=$

Along the same explanation it is possible to define the corresponding function of the remaining curves.

Careful examination of Fig. 4 shows that values of the predicted FAF (i. e, derived from a GRNN model) are obviously different from the exact ones (Labeled Target outputs), especially when only a few numbers of site parameters are considered. Note that using all site parameters will give values of the FAF close to the analytical FAF (see for instance Fig. 4 (top) for REP).

Obviously the differences between predicted and actual FAF vary from one soil profile to another. That is why it is important to analyze some statistic entity as the standard deviation of residuals, correlation coefficient, ..., which the goal is to obtain a statistically meaningful insight in the relative performances of each of the considered site proxies which control Fourier amplification factors (FAF).

Table 3 Velocity structure for the two example soil profiles SP1 (part of the REP set) and SP2 (outside TRP set).

\begin{tabular}{|c|c|c|c|c|}
\hline \multirow{2}{*}{$\begin{array}{l}\text { Layer } \\
\#\end{array}$} & \multicolumn{2}{|c|}{ Soil profile 1 (SP1) } & \multicolumn{2}{|c|}{ Soil profile 2 (SP2) } \\
\hline & $\begin{array}{c}\text { Thickness } \\
h_{i}(\mathrm{~m})\end{array}$ & $\begin{array}{c}\text { S-wave velocity } \\
V_{i}(\mathrm{~m} / \mathrm{s})\end{array}$ & $\begin{array}{c}\text { Thickness } \\
h_{i}(\mathrm{~m})\end{array}$ & $\begin{array}{c}\text { S-wave velocity } \\
V_{i}(\mathrm{~m} / \mathrm{s})\end{array}$ \\
\hline 1 & 5 & 431 & 2.5 & 252 \\
\hline 2 & Bedrock & 2253 & 2.5 & 91 \\
\hline 3 & & & 17.5 & 195 \\
\hline 4 & & & Bedrock & 800 \\
\hline
\end{tabular}

Table 4 Site parameters for the two example soil profiles SP1 (part of the REP set) and SP2 (outside TRP set).

\begin{tabular}{lcccccc}
\hline $\begin{array}{l}\text { Soil } \\
\text { profile }\end{array}$ & $\begin{array}{c}\text { Depth } \\
(\mathrm{m})\end{array}$ & $\begin{array}{c}f_{0} \\
(\mathrm{~Hz})\end{array}$ & $\begin{array}{c}V_{\text {sm }} \\
(\mathrm{m} / \mathrm{s})\end{array}$ & $C v$ & $\begin{array}{c}V_{\text {s30 }} \\
(\mathrm{m} / \mathrm{s})\end{array}$ & $\begin{array}{c}V_{\text {bedrock }} \\
(\mathrm{m} / \mathrm{s})\end{array}$ \\
\hline SP1 & 5 & 27.43 & 431 & 5.22 & 431 & 2253 \\
SP2 & 22.5 & 2.30 & 177 & 8.79 & 177 & 800 \\
\hline
\end{tabular}

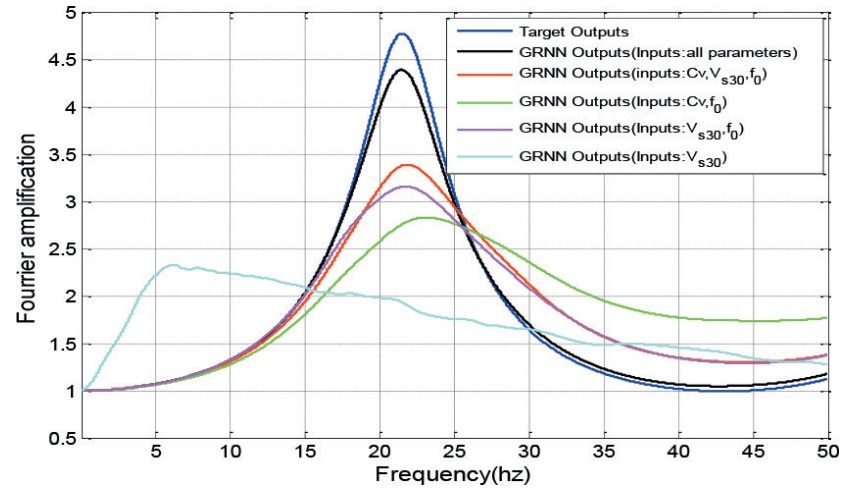

(a)

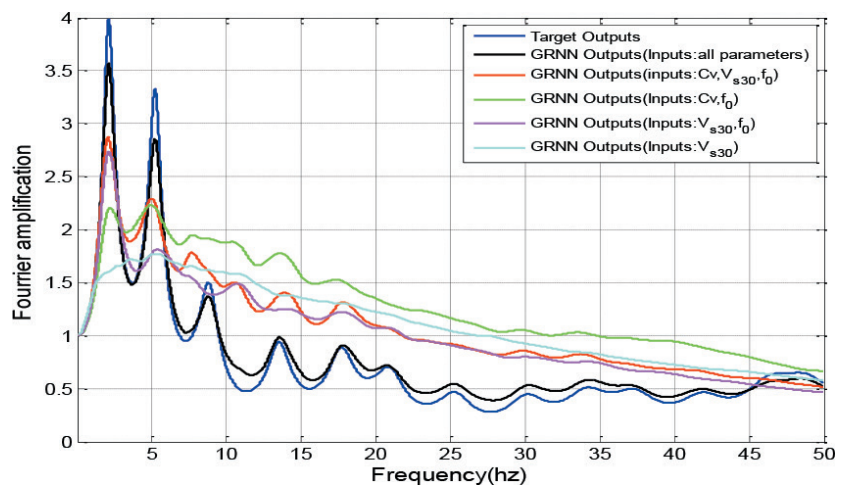

(b)

Fig. 4 Comparison between the actual FAF from analytical model and the corresponding GRNN predictions for a) a soil profile corresponding to one of the 858 to the real profiles REP (top), and b) one of the soil profiles to truncated profiles TRP (bottom)

We will not compare $\operatorname{FAF}\left(P_{k}, \theta, f_{0}\right)$ and $\operatorname{FAF}\left(P_{k}, \theta, f_{0}\right.$, $\left.x_{1}, \ldots, x_{R}\right)$ since value of $f_{0}$ will be surely close to one of the values of $f_{i}$.

\subsection{Analysis of statistic entity}

The error between prediction and actual values obtained with analytical model has been estimated with the following quantities where the goal is compared with the initial variabilities compiled by Eqs. (9-11) the new variabilities are defined by Eqs. (16-18).

- For each frequency and each GRNN model, a frequency dependent error can be defined as follow:

$$
\begin{aligned}
& \varepsilon\left(\theta, f_{i}, x_{1} \ldots x_{R}\right)= \\
& \sqrt{\frac{1}{n_{P}} \sum_{k=1}^{n_{P}}\left[\log \left(F A F\left(P_{k}, \theta, f_{i}, x_{1} \ldots x_{R}\right)\right)-\log \left(F A F\left(P_{k}, \theta, f_{i}\right)\right)\right]^{2}}
\end{aligned}
$$

- The maximum error is computed as the maximum over all frequencies of this frequency dependent error term

$$
\varepsilon_{\max }\left(\theta, x_{1} \ldots x_{R}\right)=\max \left[\varepsilon\left(\theta, f_{i}, x_{1} \ldots x_{R}\right)\right] .
$$


- Finally, a total of overall error is defined as the average over all frequencies of the error term

$$
\varepsilon_{m}\left(\theta, x_{1}, \ldots, x_{R}\right)=\frac{1}{n_{f}} \sum_{i=1}^{n_{f}} \varepsilon\left(\theta, f_{i}, x_{1}, \ldots, x_{R}\right) .
$$

Examples of the frequency dependent error $\varepsilon\left(\theta, f_{i}, x_{1}, \ldots, x_{R}\right)$, called also Standard deviation of residuals, are displayed in Fig. 5 (top) and Fig. 5 (bottom) for the real profiles (REP) and truncated profiles (TRP) respectively, together with the initial standard deviation (Eq. (10)) of the FAF. In the former case, the few considered GRNN models are the same as those considered for Fig. 4, i.e., the pairs $\left(C_{v}, f_{0}\right)$ and $\left(f_{0}, V_{s 30}\right)$ the triplet $\left(C_{v}, f_{0}, V_{s 30}\right)$ and the "all parameter" case, adding the case of a one parameter GRNN.

These plots exhibit several noticeable features:

$f_{0}$ alone allows a significant explanation of the FAF (i.e., $\varepsilon\left(\theta, f_{i}, x_{5}\right)$ is significantly smaller than $\left.\sigma\left(\theta, f_{i}\right)\right)$. When considering two site parameters, the pair $\left(f_{0}, V_{s 30}\right)$ is very good for truncated profiles and real profiles. But sometimes the pair $\left(f_{0}, C_{v}\right)$ is slightly reliable at low to intermediate frequency range for real profiles REP. This result is logical because the contrast of velocity is very important for REP then for TRP.

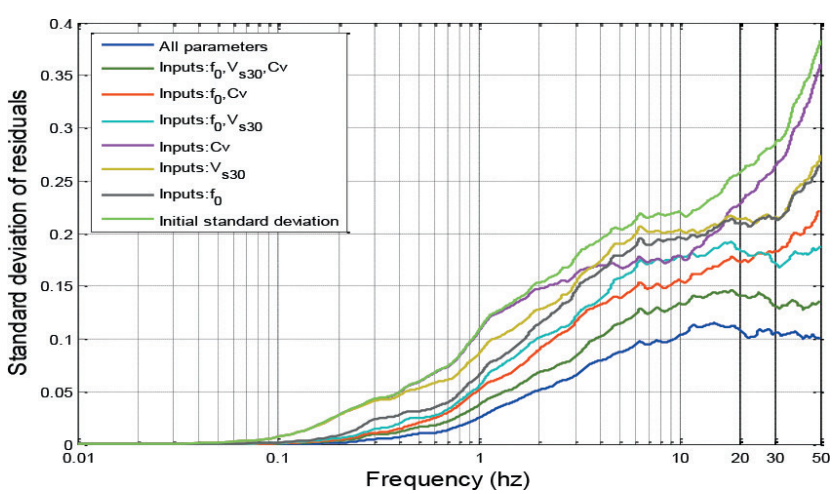

(a)

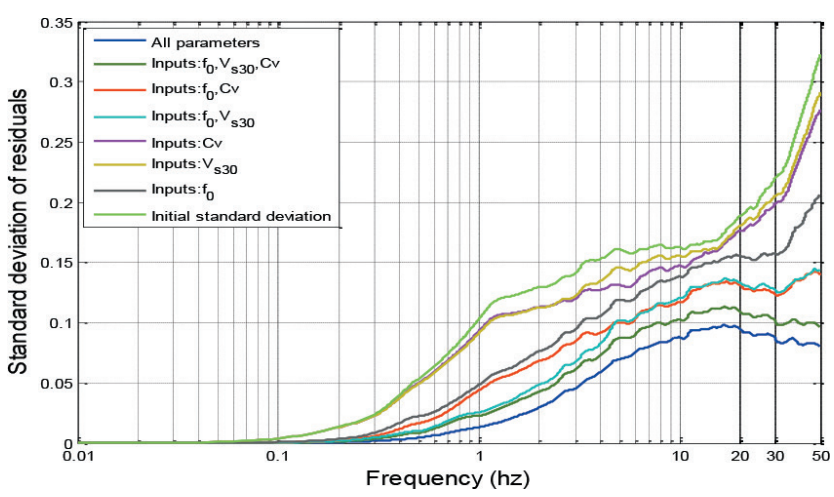

(b)

Fig. 5 Standard deviation of residuals of FAF predicted by GRNN compared to analytical model for a) REP (top) and b) TRP (bottom)
The three parameter GRNN model is very powerful to predict actual FAF, while the "all parameter" GRNN model leads to the lowest value of $\varepsilon\left(\theta, f_{i}, x_{1}, x_{2}, x_{3}, x_{4}, x_{5}, x_{6}\right)$ and which are also lower to the initial variability $\sigma\left(\theta, f_{i}\right)$.

$\varepsilon\left(\theta, f_{i}, x_{1}, \ldots, x_{R}\right)$ are maximum in the intermediate to high frequency range for the real profiles REP and TRP.

The widely used parameter $V_{s 30}$ is more efficient for TRP, i.e. when the reference bedrock velocity is the same for all profiles.

However, these results are only partial as only 6 out of the many possible models (for instance up to 63 for REP). Considering the large number of possible combinations (Table 3) we analyzed the respective performances of each proxy by evaluating, for a given number of site proxies, the average value of $\varepsilon_{m}\left(\theta, x_{1}, \ldots, x_{R}\right)$ for all the proxy combinations that involve a given proxy. For instance let consider the case of two site parameters. We want to assess the performance of the site parameter $x_{1}$. The combinations that involve this site parameters are $\left\{\left(x_{1}, x_{2}\right) ; \ldots\left(x_{1}, x_{R}\right)\right\}$. Thus we have the following value of the $\left\{\varepsilon_{m}\left(\theta, x_{1}, x_{2}\right) ; \ldots \varepsilon_{m}\left(\theta, x_{1}, x_{R}\right)\right\}$ that will be reduced to one value which is the average $\varphi_{m}\left(\theta, x_{1}, 2\right)$ defined by:

$\varphi_{m}\left(\theta, x_{1}, 2\right)=\frac{1}{R-1} \sum_{l=2}^{l=R} \varepsilon_{m}\left(\theta, x_{1}, x_{l}\right)$.

This average value will be compared to the overall initial variability through the quantity $R S_{m}\left(\theta, x_{1}, 2\right)$ which is called the Reduction of standard deviation

$R S_{m}\left(\theta, x_{1}, 2\right)=1-\frac{\varphi_{m}\left(\theta, x_{1}, 2\right)}{\sigma_{m}(\theta)}$.

In the case where we intend to measure the performance of the site parameters $x_{1}$ but with three site parameters then the average value will be $\varphi_{m}\left(\theta, x_{1}, 3\right)$. Thus Eq. (21) will be written:

$R S_{m}\left(\theta, x_{1}, 3\right)=1-\frac{\varphi_{m}\left(\theta, x_{1}, 3\right)}{\sigma_{m}(\theta)}$.

This approach could be generalized when we intend to measure the performance of a given site parameters $x_{l} ;\{l=1, \ldots, R\}$ if we consider NPR site parameters (with $\{N P R=2, \ldots, R\})$. Thus the Reduction of standard deviation will be:

$R S_{m}\left(\theta, x_{l}, N P R\right)=1-\frac{\varphi_{m}\left(\theta, x_{l}, N P R\right)}{\sigma_{m}(\theta)}$.

For the case of $N P R=1$, the Reduction of standard deviation is written: 
$R S_{m}\left(\theta, x_{l}, 1\right)=1-\frac{\varepsilon_{m}\left(\theta, x_{l}\right)}{\sigma_{m}(\theta)}$.

Globally, if $R S_{m}\left(\theta, x_{l}, N P R\right)$ tends to zero then the site paramaters $x_{l}$ do not perform very well.

Another entity can be used, it defined as the variance reduction, the following equation can be expressed such as:

$R \operatorname{var}_{m}\left(\theta, x_{l}, N P R\right)=1-\left(\frac{\varphi_{m}\left(\theta, x_{l}, N P R\right)}{\sigma_{m}(\theta)}\right)^{2}$.

Fig. 6 displays the evolution of $R S_{m}\left(\theta, x_{l}, N P R\right)$ with NPR for a given site parameters $x_{l}$. While it clearly decreases with an increasing number of explanatory site proxies, it also exhibits a significant scatter for a given number of explanatory site proxies: this indicates that some site proxies perform better than other in controlling the FAF.

Let analyze the results obtained for the case of one site parameter i.e $R S_{m}\left(\theta, x_{l}, 1\right)$. Fig. 6 shows that there is one parameter which performs systematically better than the others to predict the FAF: The resonant frequency $\left(f_{0}\right)$ that controls the actual Fourier amplification. The remaining parameters perform almost similarly. To some extent the average shear wave velocity of the upper $30 \mathrm{~m}\left(V_{s 30}\right)$ has a better performance for REP (Fig. 6 (top)), while the site parameter Depth is somehow better for TRP (Fig. 6 (bottom)).

Consider now the case of two site parameters. Careful examination of Fig. 6 show that again the resonant frequency $\left(f_{0}\right)$ performs better than the other site parameters. However, for both REP and TRP it is clear that the other site parameters exhibit similar value of the Reduction Standard Deviation.

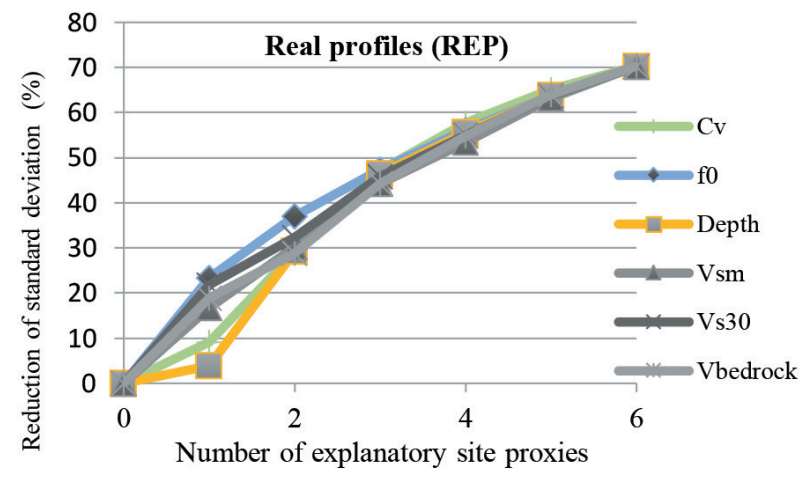

(a)

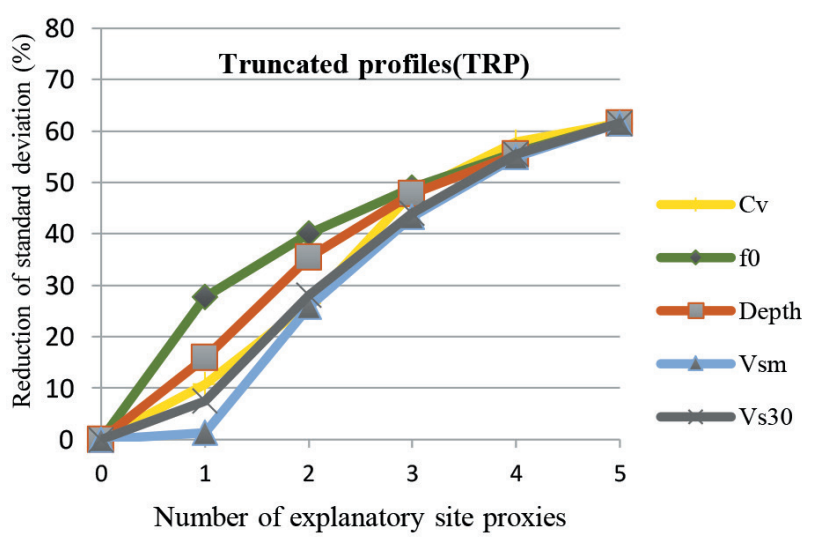

(b)

Fig. 6 Illustration of reduction of the standard deviation of residuals between GRNN models and actual FAF with the number of site proxies considered model for a) REP (top) and b) TRP (bottom)

The question that arises here: what is the optimal choice? Careful examination of Table 5 indicates an error reasonable for the pair $\left(f_{0}, V_{s 30}\right)$. This results are very interesting for REP and TRP (see Table 5) these cases that indicate clearly the variance reduction is $61 \%$ for REP and

Table 5 Standard deviation of model residuals for various GRNN models implying the initial, actual frequency Fourier amplification factors (REP, TRP cases, last two columns) for various combinations of site parameters

\begin{tabular}{|c|c|c|c|}
\hline Number of parameters & Considered site parameters & $\begin{array}{l}\text { Standard deviation for real } \\
\text { profiles(REP) }\end{array}$ & $\begin{array}{c}\text { Standard deviation for truncated } \\
\text { profiles (TRP) }\end{array}$ \\
\hline All (6) for REP All(5) for TRP & Depth $+f_{0}+V_{s m}+C_{v}+V_{s 30}+V_{\text {bedrock }}$ & 0.0828 & 0.0841 \\
\hline 4(best quadruplet) & Depth $+f_{0}+C_{v}+V_{s 30}$ & 0.1083 & 0.0883 \\
\hline 3 (best triplet) & $f_{0}+C_{v}+V_{s 30}$ & 0.1306 & 0.0993 \\
\hline 2 (best pair) For TRP & $f_{0}+C_{v}$ & 0.1775 & 0.1251 \\
\hline 2 (convenient pair) & $f_{0}+V_{s 30}$ & 0.1740 & 0.1262 \\
\hline 2 (best pair) for REP & $f_{0}+V_{s m}$ & 0.1717 & 0.1321 \\
\hline 1 & $C_{v}$ & 0.2532 & 0.1955 \\
\hline 1 (usual) & $V_{s 30}$ & 0.2181 & 0.2026 \\
\hline 1 (best) & $f_{0}$ & 0.2137 & 0.1581 \\
\hline \multicolumn{2}{|c|}{ Overall Initial variability term $\sigma_{m}(\theta)$} & 0.2788 & 0.2189 \\
\hline
\end{tabular}


$67 \%$ for TRP but that indicate $41 \%$ for REP and $48 \%$ for TRP when considering $f_{0}$ directly as an input parameter (best parameters). The latter procedure is however simple, and allows to have less complex prediction formula.

It desirable also to derive a relationship between the FAF derived from the GRNN and some site proxies. As the FAF depends on the frequency, we will focus only on the resonant frequency.

\subsection{Variation of FAF at resonance frequency using GRNN}

In this section, we will focus on the establishment of a relationship between the FAF at a resonance frequency and a number of site parameters through the GRNN. From an engineering point of view, TRP are more interesting than REP since for this latter the velocity at bedrock exceeds largely $800 \mathrm{~m} / \mathrm{s}$ which is the most accepted value. For this purpose the relationship between FAF and site parameters will be established only for TRP. However, we can easily establish this relationship for REP. Regarding the number of site parameters, the best situation is to establish a relationship between FAF and all site parameters because this situation will give a good prediction of the FAF (see for instance Fig. 4 and Fig. 6). However from an engineering point of view this situation is not desirable. Along this idea it is more suitable to use one or at least two site parameters.
Which site parameters have to be used? The best approach to choose the site parameters is to compute the overall error of the FAF (see Eq. (19)). Note that this overall error does not depends on the frequency since this latter is equal to $f_{0}$.

Table 6 provides values for different cases. Evidently the case of all site parameters ( 6 for REP and 5 for TRP) leads to the lowest value of the standard deviation. Alternatively the highest values of the standard deviation are obtained when we introduce only one site parameter.

Note that for TRP the best site parameter is $V_{s m}$, while for REP $C_{v}$ is the best one. In the case of two site parameters, results show that for TRP the best pair is $\left(f_{0}, V_{s m}\right)$ while for REP the best pair is (Depth, $C_{v}$ ). These results show that for $C_{v}$ is predominant for REP and $V_{s m}$ is predominant for TRP. Both site parameters are however very hard to obtain in real situation. The best convenient pair is $\left(f_{0}, V_{s 30}\right)$ especially for TRP.

On the other hand, this relationship has been established for soil profile that has values of site parameters $\left(f_{0}, V_{s 30}\right)$ within the distribution of site parameters (see for instance: Boudghene Stambouli et al. [12]).

With these assumptions the FAF will be written $\operatorname{FAF}\left(f_{0}, V_{s 30}\right)$.

Through the GRNN method the following relationship has been established

Table 6 Standard deviation of model residuals for various GRNN models for Fourier amplification factor at resonance frequency (REP, TRP cases, last two columns) for various combinations of site parameters

\begin{tabular}{|c|c|c|c|c|c|}
\hline $\begin{array}{l}\text { Number of } \\
\text { parameters }\end{array}$ & Considered site parameter & $\begin{array}{l}\text { Standard deviation for } \\
\text { real profiles (REP) at } \\
\text { FAF }\left(f_{0}\right)\end{array}$ & $\begin{array}{l}\text { Correlation } \\
\text { Coefficient } \\
\qquad\left(\mathrm{R}^{2}\right)\end{array}$ & $\begin{array}{l}\text { Standard deviation } \\
\text { for truncated profiles } \\
(\mathrm{TRP}) \text { at } \mathrm{FAF}\left(f_{0}\right)\end{array}$ & $\begin{array}{c}\text { Correlation } \\
\text { Coefficient } \\
\qquad\left(\mathrm{R}^{2}\right)\end{array}$ \\
\hline $\begin{array}{l}\text { All (6) for REP } \\
\text { All (5) for TRP }\end{array}$ & Depth $+f_{0}+V_{s m}+C_{v}+V_{s 30}+V_{\text {bedrock }}$ & 0.0824 & 0.93 & 0.041 & 0.96 \\
\hline 4 (best quadruplet) & Depth $+f_{0}+C_{v}+V_{s 30}$ & 0.0884 & 0.92 & 0.0454 & 0.95 \\
\hline 3 (best triplet) & $f_{0}+C_{v}+V_{s 30}$ & 0.1072 & 0.87 & 0.0567 & 0.93 \\
\hline 2 & $f_{0}+C_{v}$ & 0.1509 & 0.72 & 0.1064 & 0.68 \\
\hline 2 (convenient pair) & $f_{0}+V_{s 30}$ & 0.1686 & 0.65 & 0.0765 & 0.86 \\
\hline 2 (best pair) For TRP & $f_{0}+V_{s m}$ & 0.1888 & 0.51 & 0.0705 & 0.88 \\
\hline 2 (best pair) for REP & Depth $+C_{v}$ & 0.1357 & 0.78 & 0.0927 & 0.77 \\
\hline 1 (best)for REP & $C_{v}$ & 0.1641 & 0.66 & 0.1178 & 0.58 \\
\hline 1 (usual) & $V_{s 30}$ & 0.2154 & 0.18 & 0.1032 & 0.71 \\
\hline 1 & $f_{0}$ & 0.2060 & 0.33 & 0.1368 & 0.33 \\
\hline 1 (best)for TRP & $V_{s m}$ & 0.2137 & 0.22 & 0.0869 & 0.81 \\
\hline \multicolumn{2}{|c|}{ Overall Initial variability term } & 0.2187 & & 0.1453 & \\
\hline
\end{tabular}


$\operatorname{FAF}\left(f_{0}, V_{s 30}\right)=10^{\left(\{y\}^{\prime} *\{H\} / \Sigma H_{i}\right)}$,

$$
\left.\{H\}=\exp \left(-\left[b \sqrt{\left[\left(\left(I W_{t, 1}\right)-\log \left(f_{0}\right)\right)^{2}+\left(\left(I W_{t, 2}-\log \left(V_{S 30}\right)\right)\right)^{2}\right.}\right] \cdot\right]^{2}\right),
$$

With

$$
\{H\}=\left[\begin{array}{c}
H_{1} \\
M \\
H_{i} \\
M \\
H_{\text {final }}
\end{array}\right],
$$

where $\left\{I W_{t, 1}\right\}$ and $\left\{I W_{t, 2}\right\}$ are vectors of size $n_{P}$ consisting of the fundamental frequencies $f_{0}$ and $V_{s 30}$ values of the $n_{P}$ sample profiles, while $\{y\}$ is the weight between the hidden and output layers.

The optimal $b$ (bias) value is determined in training phase, and found to be equal to 9.25 .

This equation should be used with caution with respect of soil parameters range (for more information see Boudghene Stambouli et al. [12]).

As indicated above, we have computed the Fourier amplification factor $\operatorname{FAF}\left(f_{0}\right)$ for the GRNN model based the pair of site proxies $\left(f_{0}, V_{s 30}\right)$ which prove to be efficient. Fig. 7 display the dependence of these 2 factors as a function of $V_{s 30}$ and $f_{0}$. For all cases, this dependence is considered within the 5\%-90\% fractile range of considered explanatory parameter: the $[0.9,22 \mathrm{~Hz}]$ interval is considered for $f_{0}$ and for $V_{s 30}$ with fracile $10 \%, 50 \%$, $75 \%$ and $90 \%$ corresponding at 200, 330420 and $510 \mathrm{~m} / \mathrm{s}$ respectively.

Fourier amplification factor is found to reach the highest values basically for soft soil with low shear wave velocity upper $30 \mathrm{~m}$. But for soft soil when $f_{0}$ increases the amplification decreases. This result is interesting, it correspond to a thin layer with low shear wave velocity overcome bedrock, situation that is quite frequent in practice. For stiff soil the amplification is less important and decreases with a lower degree sometimes.

\section{Conclusions}

In Nuclear power industry application, the Fourier Amplification Factor (FAF) is mostly used from this, the goal of this study is essentially to identify the key parameters controlling Fourier Amplification Factor (FAF) using wave propagation theory and a neural network method called

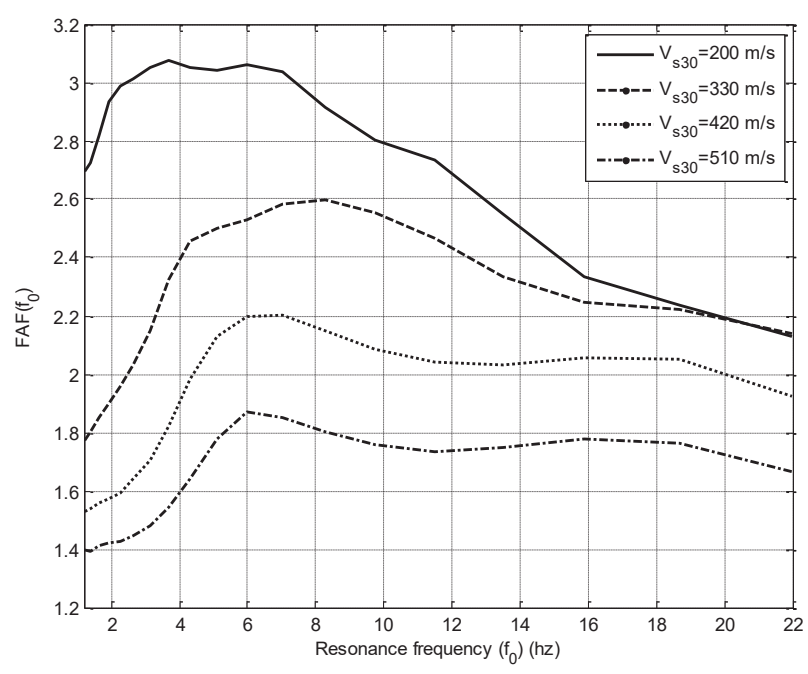

Fig. 7 Variation of Fourier amplification factor $\operatorname{FAF}\left(f_{0}\right)$ with $f_{0}$ and $V_{s 30}$

GRNN. First, we have derived a set of FAF in linear viscous elastic domain for 858 real sites. The Fourier amplification factor were derived from the module of transfer function, both in terms of frequency dependent Fourier amplification factors $\operatorname{FAF}(f)$, and of "summary" $\operatorname{FAF}\left(f_{0}\right)$ which is correspondent to the amplification factors at resonance frequency. Second, GRNN (Generalized Regression Neural Network) models were used to investigate the relationship between these amplification factors and several "usual" site proxies, i.e., $V_{s 30}, f_{0}$, sediment thickness, the maximum velocity contrast and bedrock velocity, the corresponding harmonic average sediment velocity.

The 858 real site profile (named REP), exhibit a large site-to-site variability of the bedrock velocity. Thus we derived from the REP a new set of soil profile, named Truncated soil profile (TRP) for which we consider that the shear wave velocity at bedrock do not exceed $800 \mathrm{~m} / \mathrm{s}$. This assumption is in accordance with many regulation codes. Many GRNN models were considered in each case, with all possible combinations of site proxies, from one single proxy to all of them considered together, which allow comparing the respective performances of every proxy to explain (and predict) site Fourier amplification.

The results show that by far, using the six site proxies lead to a better prediction of FAF. However, from a practical view it is desirable to have only one or at least site proxies to use for the assessment of the FAF. The results show that the best performing single site parameter is the overall resonance frequency $f_{0}$. This site parameters is easy to assess through, for instance, through the H/V method developed by Nakamura [38]. However one single site parameter is not insufficient to predict the Fourier 
amplification. Other more convenient parameters are preferred, among which the couple $\left(V_{s 30}, f_{0}\right)$ which is shown to allow reducing the variance of residuals by at least $61 \%$ for REP and $67 \%$ for (TRP). This result is very useful for the application in Nuclear Power Industry.

In a code perspective, equation for Fourier amplification factor corresponding at resonance frequency for any sites has been established.

The main result of this paper are very interesting for evaluation where proxies give the best result on Fourier amplification ratio and can be used for low seismicity areas respecting the use of the1D linear viscoelastic (i.e. for PGA inferior at $0.2 \mathrm{~g}$ ) (see for instance: Trifunac and Todorovska [22] among others) which explains the limitation of the 1D linear visco-elastic.

\section{References}

[1] Trifunac, M. D. "How to model amplification of strong earthquake motions by local soil and geologic site conditions", Earthquake Engineering and Structural Dynamics, 19(6), pp. 833-846, 1990. https://doi.org/10.1002/eqe.4290190605

[2] Thomson, W. T. "Transmission of Elastic Waves through a Stratified Solid Medium", Journal of Applied Physics, 21(2), pp. 89-93, 1950. https://doi.org/10.1063/1.1699629

[3] Haskell, N. A. "The dispersion of surface waves on multilayered media", Bulletin of the Seismological Society of America, 43(1), pp. 17-34, 1953.

[4] Biro, Y., Renault, P. "Importance and Impact of Host-to-Target Conversions for Ground Motion Prediction Equations in PSHA", [pdf] presented at the 15th World Conference on Earthquake Engineering, Lisbon, Portugal, Sept. 24-28, 2012. Available at: https:/www.iitk.ac.in/nicee/wcee/article/WCEE2012_1855.pdf

[5] Renault, P. L. A., Abrahamson, N. A., SP3 Experts "PEGASOS Refinement Project, Volume 5 SP3 - Site Response Characterization - Evaluation Summaries and Hazard Input Documents", Swissnuclear, Olten, Switzerland, 2014. [online] Available at: https://www.swissnuclear.ch/de/search.html?searchtext=Site + Response+Characterization

[6] Reed, J. W., Anderson, N., Chokshi, N. C., Kennedy, R. P., Metevia, W. J., Ostrom, D. K., Stevenson, J. D. "A criterion for determining exceedance of the operating basis earthquake", Electric Power Research Institute, Palo Alto, CA, USA, Rep. EPRI-NP-5930, 1988.

[7] US Nuclear Regulatory Commission "Regulatory Guide 1.166 Pre-earthquake planning and immediate nuclear power plant operator postearthquake actions", [pdf] Office of Nuclear Regulatory Research, Washington, DC, USA, 1997. Available at: https:/www. nrc.gov/docs/ML0037/ML003740089.pdf

[8] Ktenidou, O.-J., Abrahamson, N. A. "Empirical Estimation of HighFrequency Ground Motion on Hard Rock", Seismological Research Letters, 87(6), pp. 1465-1478, 2016. https://oi.org/10.1785/0220160075
However an important next step will be to consider for computing a new Fourier amplification factor using nonlinear site response method. Where the characteristics of different layers of each soil profile are different. However they use a degradation curve for each layer such a curve developed by Darendeli [39]. In this step the Fourier amplification factor become dependent on seismic signal in contrary to linear approach.

\section{Acknowledgement}

This work has been partially supported by the project: "Prédiction du mouvement sismique et estimation du risque sismique lié aux effets de site" 13MDU901 Tassili CMEP between Universities of Tlemcen (Algeria) and Grenoble (France).

[9] Bard, P.-Y., Bora, S. S., Hollender, F., Laurendeau, A., Traversa, P. "Are the Standard VS-Kappa Host-to-Target Adjustments the Only Way to Get Consistent Hard-Rock Ground Motion Prediction?", Pure and Applied Geophysics, 177, 2019. https://doi.org/10.1007/s00024-019-02173-9

[10] 1997 Uniform Building CodeTM "Structural Engineering Design Provisions", International Conference of Building Officials, Whittier, CA, USA, 1997.

[11] CEN "Eurocode 8: Design of structures for earthquake resistance - Part 1: General rules, seismic actions and rules for buildings", European Committee for Standardization, Brussels, Belgium, 2005.

[12] Boudghene Stambouli, A., Zendagui, D., Bard, P.-Y., Derras, B. "Deriving amplification factors from simple site parameters using generalized regression neural networks: implications for relevant site proxies", Earth, Planets and Space, 69, Article number: 99, 2017. https://doi.org/10.1186/s40623-017-0686-3

[13] Andrews, D. J. "Objective Determination of Source Parameters and Similarity of Earthquakes of Different Size", Earthquake Source Mechanics, 37, pp. 259-267, 1986.

https://doi.org/10.1029/GM037p0259

[14] Boatwright, J., Seekins, L. C., Fumal, T. E., Liu, H.-P., Mueller, C. S. "Ground motion amplification in the Marina District", Bulletin of the Seismological Society of America, 81(5), pp. 1980-1997, 1991.

[15] Hartzell, S. "Variability of Site Response in Seattle, Washington", Bulletin of the Seismological Society of America, 90(5), pp. 12371250, 2000. https://doi.org/10.1785/0120000022

[16] Sokolov, V. Y. "Empirical models for estimating Fourier-amplitude spectra of ground acceleration in the northern Caucasus (Racha seismogenic zone)", Bulletin of the Seismological Society of America, 87(6), pp. 1401-1412, 1997.

[17] Sokolov, V., Loh, C.-H., Wen, K.-L. "Empirical model for estimating Fourier amplitude spectra of ground acceleration in Taiwan region", Earthquake Engineering and Structural Dynamics, 29(3), pp. 339-357, 2000. https://doi.org/10.1002/(SICI)1096-9845(200003)29:3<339::AIDEQE908>3.0.CO;2-R 
[18] Bora, S. S., Scherbaum, F., Kuehn, N., Stafford, P. "Fourier spectral-and duration models for the generation of response spectra adjustable to different source-, propagation-, and site conditions", Bulletin of Earthquake Engineering, 12(1), pp. 467-493, 2014.

[19] Bora, S. S., Scherbaum, F., Kuehn, N., Stafford, P. "Development of a Response Spectral Ground-Motion Prediction Equation (GMPE) for Seismic-Hazard Analysis from Empirical Fourier Spectral and Duration Models", Bulletin of the Seismological Society of America, 105(4), pp. 2192-2218, 2015. https://doi.org/10.1785/0120140297

[20] Bora, S. S., Scherbaum, F., Kuehn, N., Stafford, P. "On the Relationship between Fourier and Response Spectra: Implications for the Adjustment of Empirical Ground-Motion Prediction Equations (GMPEs)", Bulletin of the Seismological Society of America, 106(3), pp. 1235-1253, 2016.

[21] O'Connell, D. R. H. "Replication of Apparent Nonlinear Seismic Response with Linear Wave Propagation Models", Science, 283(5410), pp. 2045-2050, 1999. https://doi.org/10.1126/science.283.5410.2045

[22] Trifunac, M. D., Todorovska, M. I. "Can aftershock studies predict site amplification factors? Northridge, CA, earthquake of 17 January 1994", Soil Dynamics and Earthquake Engineering, 19(4), pp. 233251, 2000.

https://doi.org/10.1016/S0267-7261(00)00011-7

[23] Cherid, D., Hammoutene, M., Tiliouine, B., Berrah, M. K. "Local seismic site amplification: effects of obliquely incident antiplane motions", Journal of Seismology, 21, pp. 509-524, 2017. https://doi.org/10.1007/s10950-016-9613-5

[24] Schnabel, P. B. "SHAKE A Computer Program for Earthquake Response Analysis of Horizontally Layered Sites", University of California, Berkeley, CA, USA, Rep. EERC 72-12, 1972.

[25] Bardet, J. P., Ichii, K., Lin, C. H. "EERA: A Computer Program for Equivalent-linear Earthquake site Response Analyses of Layered Soil Deposits", University of Southern California, Los Angeles, CA, USA, 2000

[26] Hashash, Y. M. A., Groholski, D. R., Phillips, C. A., Park, D., Musgrove, M. "DEEPSOIL 5.1 User Manual and Tutorial", Department of Civil and Environmental Engineering, University of Illinois at Urbana-Champaign, Urbana, IL, USA, 2012.
[27] Kramer, S. L. "Geotechnical Earthquake Engineering", PrenticeHall, NJ, USA, 1996.

[28] Aki, K., Richards, P. G. "Quantitative seismology, theory and methods", 1st ed., WH Freeman \& Co., New York, NY, USA, 1980.

[29] Fukushima, Y., Gariel, J.-C., Tanaka, R. "Site-Dependent Attenuation Relations of Seismic Motion Parameters at Depth Using Borehole Data", Bulletin of the Seismological Society of America, 85(6), pp. 1790-1804, 1995.

[30] Di Giulio, G., Savvaidis, A., Ohrnberger, M., Wathelet, M., Cornou, C.,..., Bard, P.-Y. "Exploring the model space and ranking a best class of models in surface-wave dispersion inversion: Application at European strong-motion sites", Geophysics, 77(3), pp. B147-B166, 2012. https://doi.org/10.1190/geo2011-0116.1

[31] Specht, D. F. "A general regression neural network", IEEE Transactions on Neural Networks, 2(6), pp. 568-576, 1991.

[32] Kim, B., Lee, D. W., Park, K. Y., Choi, S. R., Choi, A. "Prediction of plasma etching using a randomized generalized regression neural network", Vacuum, 76(1), pp. 37-43, 2004.

[33] Nadaraya, E. A. "On estimating regression", Teoriya Veroyatnostei i ee Primeneniya, 9(1), pp. 157-159, 1964.

[34] Watson, G. S. "Smooth regression analysis", Sankhyā: The Indian Journal of Statistics, Series A, 26(4), pp. 359-372, 1964.

[35] Parzen, E. "On Estimation of a Probability Density Function and Mode", Annals of Mathematical Statistics, 33(3), pp. 1065-1076, 1962.

[36] Benahmed, B., Hamoutenne, M. "Use of the Artificial Neural Networks to Estimate the DRF for Eurocode 8", Periodica Polytechnica Civil Engineering, 62(2), pp. 470-479, 2018. https://doi.org/10.3311/PPci.8139

[37] Wasserman, P. D. "Advanced methods in neural computing", John Wiley \& Sons, New York, NY, USA, 1993.

[38] Nakamura, Y. "A method for dynamic characteristics estimation of subsurface using microtremor on the ground surface", Railway Technical Research Institute, Quarterly Reports, 30(1), pp. 25-33, 1989.

[39] Darendeli, M. B. "Development of a new family of normalized modulus reduction and material damping curves", $\mathrm{PhD}$ Thesis, The University of Texas at Austin, 2001. 